\title{
RESEÑ $A$ \\ El Dao de la sabiduría, de Filippo Costantini. Puntarenas: Editorial Sede del Pacífico, 2021. 378 páginas. ISBN 978-9930-9732-1-9
}

\section{REVIEW \\ El Dao de la sabiduria by Filippo Costantini. Puntarenas: Editorial Sede del Pacífico, 2021. 378 pages. ISBN 978-9930-9732-1-9}

JOSÉ ANTONIO CERVERA JIMÉNEZ

Centro de Estudios de Asia y África, El Colegio de México, Ciudad de México, México jacervera@colmex.mx

Resumen: La editorial de la Sede del Pacífico de la Universidad de Costa Rica acaba de publicar una gran obra sobre filosofía china. Se trata de El Dao de la sabiduria, del investigador italiano afincado en Costa Rica, Filippo Costantini.

Abstract: The publishing house Editorial Sede del Pacífico, of the University of Costa Rica, has just published a great work on Chinese philosophy. It is El Dao de la Sabiduria, by the Italian researcher based in Costa Rica, Filippo Costantini. 
Citar como: Cervera-Jiménez, J. A. (2022). Reseña del libro «El Dao de la sabiduría», de Filippo Costantini. Puntarenas: Editorial Sede del Pacífico, 2021. 378 páginas. ISBN 978-9930-9732-1-9. Revista Internacional de Estudios Asiáticos, 1(1), 226-233.

Fecha de recepción: 08-11-2021 | Fecha de aceptación: 09-11-2021 
La editorial de la Sede del Pacífico de la Universidad de Costa Rica acaba de publicar una gran obra sobre filosofía china. Se trata de El Dao de la sabiduría, del investigador italiano afincado en Costa Rica, Filippo Costantini.

El libro se centra en el daoísmo. La corriente daoísta incluye un conjunto muy amplio de textos y de prácticas. No he querido escribir "corriente filosófica daoísta”, porque, para empezar, el daoísmo no se puede considerar solamente como una filosofía, sino también como una religión. En China nunca hubo una dicotomía clara entre "lo filosófico" y "lo religioso". Fue sobre todo en Occidente donde se quiso establecer una diferenciación clara entre un daoísmo filosófico y un daoísmo religioso. Los eruditos occidentales se centraron más en el daoísmo filosófico y se consideró la tendencia más religiosa como supersticiosa, como de un "nivel más bajo”. En realidad, no se puede separar entre filosofía y religión. El daoísmo agrupa un enorme conjunto de manifestaciones: algunas que calificaríamos claramente como religiosas, otras como más filosóficas (hay un conjunto extensísimo de textos), y hay también una gran cantidad de prácticas (relacionadas con la salud, por ejemplo). La idea de que no se puede separar el daoísmo filosófico del daoísmo religioso subyace a lo largo de este libro.

En su obra, Costantini realiza un trabajo impresionante, se centra en fuentes primarias y utiliza también numerosas fuentes secundarias. Este libro que tenemos ante nosotros es extraordinariamente complejo, profuso en sus detalles. No es precisamente una "historia mínima" o "breve" del daoísmo. Muy detallista en las explicaciones, en la investigación, se ve que el autor ha leído muchísimo, realizando un trabajo muy erudito. Parece una tesis doctoral. No es precisamente un libro para llevarse de vacaciones a la playa. Este libro lo aconsejo para los que realmente quieran entender, de manera muy erudita, el daoísmo, y no solo el daoísmo, sino la filosofía china en su conjunto. Esta obra va mucho más allá del daoísmo. El Dao de la sabiduría proporciona una información muy detallada sobre cómo fue entendido el Dao por varias corrientes de pensamiento en China, y en general, sobre la cosmovisión china a lo largo de los siglos.

Este libro de Costantini se centra en dos cuestiones: el Dao 道, uno de los conceptos fundamentales de la filosofía china a lo largo de toda la 
historia, y la sabiduría, o más bien, el papel del sabio en el mundo y en la sociedad. El texto examina el famoso libro de Laozi 老子, el Daodejing 道德經, uno de los más importantes de ese conjunto de ideas y doctrinas que agrupamos con el nombre de "daoísmo". Hay decenas, cientos de lecturas del texto, pero Costantini se centra en tres de ellas, tres lecturas diferentes del Daodejing.

Una lectura temprana relacionada con el cultivo de la materia o energía vital, el qi 氣, es el Heshang gong 河上公. Este texto ha sido categorizado muchas veces como un texto religioso. Pero, como ya he señalado, y como muestra muy bien Costantini, no podemos circunscribir este comentario a nuestra estrecha concepción occidental de "religión". De hecho, este texto puede ser considerado como un manual práctico para la élite gobernante en la corte.

El Heshang gong es un texto relacionado con la corriente Huang-Lao 黃老, muy importante en China desde mitad del periodo de los Reinos Combatientes hasta el siglo II a.n.e. En el movimiento Huang-Lao hay tres orientaciones principales: la cosmología, que es la base del sistema, las técnicas de autocultivo que definen la dimensión psicológica (o psicofísica, como apunta Costantini) y el pensamiento político, o vía del buen gobierno.

En el Heshang gong, el Dao se relaciona directamente con el mundo natural. El Dao se puede conocer y puede guiar al ser humano. El sabio es un modelo de hombre para todos los seres humanos. En ese sentido, se parece al ideal de sabio de los confucianos. En la página 238, Costantini afirma: "Uno de los aspectos principales del comentario [el Heshang gong] es el paralelismo entre la cultivación personal y el arte del buen gobierno, los cuales no solo deben estar estrechamente relacionados, sino que debe depender uno del otro". La cosmología subyacente es correlativa, siguiendo el principio dual del yin y el yang y una correspondencia entre el Cielo, la Tierra y el ser humano. El gobernante ideal gobierna sin actuar, wu wei 無為, uno de los conceptos fundamentales del daoísmo. En definitiva, como dice Costantini en la página 61, citando a Robinet, "para Heshang gong, el Daodejing es un manual de arte de gobierno y, al mismo tiempo, de técnicas por prolongar la vida”. 
El segundo comentario estudiado por Costantini es el Xiang Er 想爾. Este constituye una lectura religiosa relacionada con instituciones daoístas. Se relaciona con una tradición religiosa surgida a finales de la dinastía Han. "El Xiang Er, de hecho, se puede definir como el primer intento de leer el Laozi como un texto sagrado, revelación de la palabra divina. Los profetas, y probablemente autores del comentario, son los fundadores del movimiento religioso daoísta llamado los Maestros Celestiales" (p. 65). Para los Maestros Celestiales, muy influyentes hacia finales de la dinastía Han, el Daodejing es un texto sagrado. El Dao no es algo oscuro e insondable, sino que se puede conocer, y llega a ser considerado como una especie de divinidad antropomorfa relacionada con el personaje de Laozi, como señala Costantini en la página 118. Hay una serie de prácticas que fueron tomadas por los Maestros Celestiales para alargar la vida.

El Xiang Er considera al Dao no como algo oscuro, sino como un principio normativo para que la gente actúe, para alargar la vida y para alcanzar la sabiduría. El ideal es conseguir la inmortalidad. Al mismo tiempo, el ideal de "sabio" es similar al que tenían los miembros de la escuela confuciana: "El Xiang Er, entonces, realiza una síntesis entre las virtudes confucianas y la tradición daoísta del Laozi. Los preceptos del Dao son totalmente morales debido a que se construyen sobre los principios claves del confucianismo como benevolencia y justicia. Además, estos principios encuentran su realización completa en la espontaneidad y en la no intencionalidad del individuo, ya que las acciones morales deliberadas son un síntoma de un declive de la sociedad que ya no lo tiene como fundamento" (p. 276). En resumen, el Xiang Er es un texto que pretende ser una guía para el autocultivo, para lograr la buena salud, la longevidad y la inmortalidad.

Por último, el comentario de Wang Bi constituye una lectura filosófico-política. Como señala el propio Costantini, "En general, el comentario de Wang Bi se considera la interpretación canónica del Laozi desde la época Song, y la principal referencia en los estudios del texto para la sinología occidental (...) El interés de Wang, de hecho, no es la especulación cosmológica o las técnicas de cultivo como en los dos anteriores y en la tradición exegética Han; Wang lee el Laozi como un texto coherente que intenta revelar la base indispensable de la realidad. Por esta razón, Laozi 
se vuelve comparable, o casi, a los otros Clásicos del antiguo pensamiento confuciano" (p. 74).

Es decir, Wang Bi no se preocupa tanto de las prácticas de autocultivo, o de llegar a la inmortalidad (como proponía el Xiang Er), ni tampoco de cuestiones cosmológicas, numerológicas, no tiene nada que ver con la cosmología correlativa que había influido tanto, por ejemplo, en el Heshang Gong. El comentario de Wang Bi es una lectura principalmente filosófica, escrita en la época Wei-Jin, un momento de gran efervescencia filosófica, de diálogo. Es la época de la Xuanxue 玄學, la Escuela del Misterio.

En el comentario de Wang Bi, el Dao se vuelve más oscuro e insondable que en los comentarios anteriores. Es importante la idea del vacío como generador. El wu 無 (“no haber") define que todo se pueda dar. En cuanto a la figura del sabio, es central para que haya paz y armonía en el imperio. El sabio está en el mundo, pero no se deja agobiar por los eventos del mundo. El sabio puede cultivarse interiormente, puede actuar en el mundo, pero sin que eso le afecte. Actúa naturalmente, aquí aparece el famoso wu wei, que no es propiamente "no actuar", sino actuar con tranquilidad y naturalidad. En resumen, el comentario de Wang Bi es más filosófico que los dos anteriores. El Dao es más oscuro que en el Heshang Gong y en el Xue Er, pero a la vez es casi confuciano. Por eso influyó mucho en los letrados confucianos, particularmente a partir de la eclosión del neoconfucianismo, durante la dinastía Song.

El Dao de la Sabiduría se divide en tres grandes capítulos. El primero se dedica a los tres comentarios y sus tradiciones. En los otros dos, Costantini se centra en dos grandes temas que se encuentran en los comentarios y que, de hecho, forman parte medular de todas las corrientes filosóficas chinas: el concepto del Dao y el papel del ser humano en el mundo.

¿Qué es el Dao? Esto es un problema. Cualquier cosa que se diga sobre el Dao no es el auténtico Dao, como proclama el famoso comienzo del Daodejing: “Dao ke dao feichang dao 道可道非常道” (“El Dao del que se puede hablar no es el Dao verdadero"). A pesar de ello, durante siglos se ha intentado dilucidar el auténtico sentido del Dao. El capítulo 2 de El Dao de la sabiduría se dedica por entero al concepto del Dao en los tres comentarios: el Heshang Gong, el Xiang Er y el comentario de Wang 
Bi. Constantini afirma que "se puede resumir el concepto de Dao en dos macrocategorías: una cosmológico-metafísica, y una normativa. Bajo la primera categoría entran las ideas del Dao como principio generativo y modelo universal. Dentro de la segunda, Dao es principio normativo porque muestra la forma en la que el ser humano necesita actuar en conformidad con sus preceptos" (p. 83).

La segunda categoría, el Dao como principio normativo, es un significado más familiar para los confucianos. El propio Confucio hablaba del Dao, el "Camino de la vida", cómo debemos actuar (y eso lleva al otro gran tema del libro de Costantini, el papel del ser humano o el "Camino del sabio"). La consideración cosmológica, el Dao como principio metafísico, es algo más propio del daoísmo que de otras corrientes, como el confucianismo, aunque sería retomado siglos después por los neoconfucianos, que llamarían a ese principio de diferentes formas, por ejemplo, Zhou Dunyi y a partir de él, Zhu Xi, lo llamarían taiji. Sin embargo, resulta que de nuevo nos encontramos en una dicotomía impuesta por los occidentales a una realidad mucho más holística en el universo del pensamiento chino. Costantini señala que, en realidad, el Dao para Confucio y para Laozi no se diferencian tanto.

El capítulo 3 del libro se dedica al estudio del papel del ser humano en el mundo, o más específicamente, a la sabiduría. Se trata de ver qué debe hacer un ser humano para llegar a su máxima potencialidad. En el fondo, este es el corazón de la ética. Costantini afirma que "Entre las cuestiones cruciales en la historia del pensamiento chino, de hecho, la naturaleza del sabio parece ser fundamental. ¿Es la sabiduría innata o se puede adquirir? ¿Es el sabio diferente de otros seres humanos o hay un camino y una forma práctica accesible para todos? Estas y otras preguntas son los temas de discusión fundamentales para muchos filósofos chinos, quienes formulan una serie de formas (daos) que apuntan a alcanzar, o por lo menos se orientan hacia la meta final. Dichas prácticas van desde la cultivación moral, hasta el arte del buen gobierno, toman en cuenta el ejercicio físico, la nutrición del espíritu, el aprendizaje intelectual y práctico” (p. 172).

Uno de los libros de historia de la filosofía clásica china más destacado es el de Angus Graham, El Dao en disputa (FCE, 2012). En él, el autor afirma que, si la pregunta crucial para los filósofos griegos y, a partir de 
ellos, los europeos de la modernidad, fue: “¿Qué es verdad?”, por el contrario, para los filósofos chinos, la pregunta esencial fue: “¿Dónde está el Camino?”, es decir, la manera para conducir la propia vida y para ordenar el estado. Ese Camino, esa forma de actuar, de gobernar y gobernarse a uno mismo, es lo que se conoce como Dao. Aquí se puede encontrar la relación entre los dos grandes temas estudiados por Costantini en su libro: el Dao y el papel del ser humano en el mundo. En el fondo, no se pueden separar ambos puntos. El Dao es el Camino del Cielo, la forma como todo se desarrolla en el universo (aspecto cosmológico-metafísico), y al mismo tiempo, el Camino del ser humano en el mundo (aspecto normativo). Si el hombre alinea su Camino, su Dao, al del Cielo, entonces se convierte en un sabio.

En El Dao de la sabiduría, el autor analiza la concepción del Dao en los tres comentarios al Daodejing: el Heshang Gong, el Xiang Er, y el comentario de Wang Bi, y también se compara el papel del ser humano y cómo se puede alcanzar la sabiduría en las tres tradiciones. A mí me gusta especialmente la visión de Wang Bi, en la que el sabio está en el mundo, pero no se deja agobiar por sus eventos. El sabio puede cultivarse interiormente, puede actuar, pero sin que eso le afecte. En un mundo cada vez más complejo, podemos volver al Daodejing y al comentario de Wang Bi para encontrar cierta luz. Pero este es solo uno de los comentarios a la gran obra daoísta. En el libro de Filippo Costantini, el lector va a encontrar una gran cantidad de información sobre la historia y la filosofía china, con una gran erudición. A través de numerosos fragmentos traducidos directamente del chino al español, se puede atisbar un poco del "alma china”. No puedo más que aconsejar la lectura de este libro, que sin duda va a abrir al lector a un mundo nuevo, y congratularme porque la Universidad de Costa Rica haya publicado esta obra que, estoy seguro, se va a convertir en una referencia para los estudios sobre filosofía china, particularmente sobre el daoísmo, en el mundo hispano.

\section{Referencias}

Costantini, F. (2021). El Dao de la sabiduria. Análisis y comparación de los tres comentarios más influyentes del Laozi Daodejing. Puntarenas: Editorial Sede del Pacífico, 2021. ISBN 978-9930-9732-1-9. 